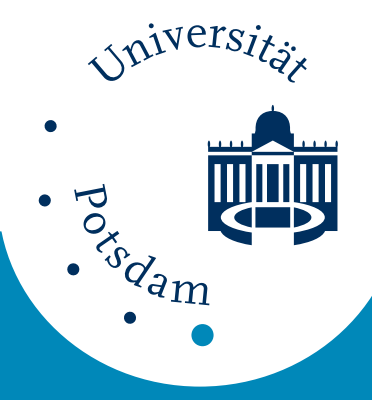

Universität Potsdam

Robert Braun, Fred Feudel, Norbert Seehafer

Bifurcations and chaos in an array of forced vortices

NLD Preprints ; 37 


\title{
Bifurcations and chaos in an array of forced vortices
}

\author{
R. Braun, F. Feudel and N. Seehafer \\ Institut für Theoretische Physik und Astrophysik, Universität Potsdam, PF 601553, D-14415 Potsdam, Germany
}

\begin{abstract}
We have studied the bifurcation structure of the incompressible two-dimensional Navier-Stokes equations with a special external forcing driving an array of $8 \times 8$ counterrotating vortices. The study has been motivated by recent experiments with thin layers of electrolytes showing, among other things, the formation of large-scale spatial patterns. As the strength of the forcing or the Reynolds number is raised the original stationary vortex array becomes unstable and a complex sequence of bifurcations is observed. The bifurcations lead to several periodic branches, torus and chaotic solutions as well as to another stationary solution. Most remarkable is the appearance of solutions characterized by structures on spatial scales large compared to the scale of the forcing. We also characterize the different dynamic regimes by means of tracers injected into the fluid. Stretching rates and Hausdorff dimensions of convected line elements are calculated to quantify the mixing process. It turns out that already for time-periodic velocity fields the mixing can be very effective.
\end{abstract}

47.20.Ky, 47.52.+j, 47.27.Cn, 47.54.+r

\section{INTRODUCTION}

The transition from simple laminar to turbulent fluid motions upon changes of parameters like the Reynolds number, though continually and intensively investigated by means of sophisticated experiments as well as analytically and numerically, is still far from being completely understood. The experiments exhibit a variety of different transition scenarios and each of them requires its own theoretical explanation.

For more than three decades efforts have been made to analyze the bifurcation behavior of a special twodimensional viscous flow, the so-called Kolmogorov flow, and to understand its transition to chaos as the Reynolds number is raised. In the Kolmogorov flow, which is produced by the forcing of just one Fourier mode, the velocity is everywhere parallel or antiparallel to a given direction perpendicular to which it varies sinusoidally. An early analytical estimation of its stability is due to Meshalkin and Sinai [1]. A recent consideration of the stability problem including linear friction and confinement effects can be found in [2].

A systematic investigation of the bifurcation structure of truncations to the two-dimensional incompressible Navier-Stokes equations (NSE) with an external forcing of the Kolmogorov type is reported in a series of papers by Franceschini and his co-workers [3-6]. Lee [7] studied a modified version of the Kolmogorov flow, generated by forcing a different Fourier mode, and found a different scenario for the transition to chaos. The flow studied by Lee has recently been systematically investigated by Feudel and Seehafer $[8,9]$, who applied special numerical bifurcation-analysis techniques.

The present paper is motivated by a number of experiments showing the formation of large-scale patterns in approximately two-dimensional fluids, apparently as the result of inverse energy cascades. So Sommeria [10] investigated the transition to turbulence produced by instabilities in a square array of electrically driven vortices. In other experiments, Tabeling et al. [11-14] studied a linear chain of forced vortices. They described the variations in the spatial structure and in the temporal evolution of the flow resulting from variations of the Reynolds number and modeled the observed phenomena by means of a one-dimensional chain of coupled oscillators. Numerical investigations based on the two-dimensional NSE and aimed at modeling these experiments were done by Finn et al. $[15,16]$. In particular with respect to the first instabilities their results show qualitative coincidences with the experimental observations.

In this paper we numerically examine the dynamics of the two-dimensional NSE with an external forcing driving an array of vortices, focussing attention on the bifurcation behavior. In Sec. II we introduce equations and forcing and briefly desribe the numerical method. Then in Sec. III we present the bifurcation scenario and explain the qualitative properties of different dynamical branches. In Sec. IV we study the Lagrangian dynamics and try to quantify the mixing process by suitable measures. Finally, Sec. V gives a short discussion of the role of the boundary conditions.

\section{BASIC EQUATIONS AND FORCING}

We study the incompressible two-dimensional NSE in the rescaled form

$$
\begin{gathered}
\frac{\partial \boldsymbol{v}}{\partial t}+(\boldsymbol{v} \cdot \boldsymbol{\nabla}) \boldsymbol{v}=\nabla^{2} \boldsymbol{v}-\nabla p+\boldsymbol{f} \\
\boldsymbol{\nabla} \cdot \boldsymbol{v}=0
\end{gathered}
$$

where $\boldsymbol{v}$ is the fluid velocity field and $p$ the thermal pressure. $\boldsymbol{f}$, the external force, is chosen to be

$$
\boldsymbol{f}=f\left(\begin{array}{r}
\sin k_{1} x \cos k_{2} y \\
-\cos k_{1} x \sin k_{2} y
\end{array}\right)
$$

Due to the rescaling of the NSE to typical length and time scales all quantities are nondimensional; the explicit 
form of the rescaling transformation may be found in Ref. [9]. The two constants in Eq. (3) are fixed to $k_{1}=$ $k_{2}=4$, so that the external force drives an array of $8 \times 8$ counterrotating eddies. For the sake of simplicity we impose periodic boundary conditions and consider the dynamics of the fluid on a square with the normalized side length $L=2 \pi$.

When using the rescaled form of the NSE with the forcing given by Eq. (3), the scalar $f$ in Eq. (3), which corresponds to the strength of the forcing, is the only free parameter and is used as bifurcation parameter. Its relation to the Reynolds number may be roughly estimated as follows. For a weak forcing the nondimensional velocity (a typical value of it) is determined by a balance between the forcing and viscous terms in Eq. (1), so that $v \approx l^{2} f$, where $l$ is the scale over which $f$ varies, $l \approx 1 / \sqrt{k_{1}^{2}+k_{2}^{2}}$. From the rescaled form of the NSE one then obtains $R e \approx l^{3} f$ for the Reynold number. For strong forcing, on the other hand, the forcing term in Eq. (1) is approximately balanced by the inertial term and one expects $R e \approx l^{3 / 2} f^{1 / 2}$ to be a better estimate.

In our numerical computations we did not directly integrate Eq. (1) but rather the corresponding equation for the vorticity $\boldsymbol{\omega}=\boldsymbol{\nabla} \times \boldsymbol{v}$. By the restriction to two spatial dimensions this equation is reduced to one scalar equation for the only nonvanishing component $\omega=\omega_{z}$ :

$$
\frac{\partial \omega}{\partial t}+(\boldsymbol{v} \cdot \nabla) \omega=\nabla^{2} \omega+f\left(k_{1}+k_{2}\right) \sin k_{1} x \sin k_{2} y
$$

For $\omega$ a Fourier expansion according to

$$
\omega=\sum_{k_{x}, k_{y} \in \mathbb{Z}} \hat{\omega}_{\boldsymbol{k}}(t) e^{i\left(k_{x} x+k_{y} y\right)}, \quad \boldsymbol{k}=\left(k_{x}, k_{y}\right),
$$

is used, leading to an infinite system of ordinary differential equations for the real and imaginary parts of the Fourier components $\hat{\omega}_{\boldsymbol{k}}$. We have numerically studied a finite-dimensional truncation of this system by means of a pseudospectral method, using a dealiased code with a resolution of $32 \times 32$ gridpoints in real space.

\section{BIFURCATION SCENARIO}

Due to the periodic boundary conditions and the form of the forcing, Eq. (1) and Eq. (4) are equivariant with respect to a special transformation group which can be represented as a semidirect sum [17] in the form

$$
G=D_{4} \dot{+}\left(Z_{4} \times Z_{4} \times Z_{2}\right),
$$

where $D_{4}$ denotes the eight-element dihedral group, $Z_{4}$ the four-element cyclic group and $Z_{2}$ the reflection group. As will be seen, the symmetry group has an essential influence on the bifurcations.

For a weak forcing, i.e. for a small value of $f$, the $8 \times 8$ array of counterrotating vortices (see Fig. 1) is the only time-asymptotic state.

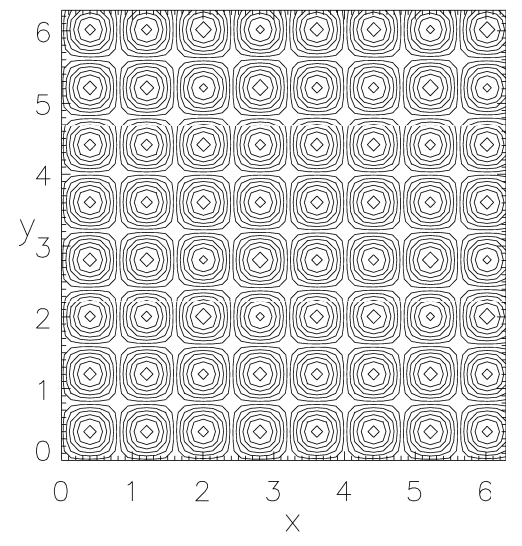

FIG. 1. Streamlines for $f=362$ (Steady I).

Here only the forced modes are excited and the vorticity can be expressed analytically in the form

$$
\omega(x, y)=\frac{f}{4} \sin (4 x) \sin (4 y) .
$$

This steady solution is symmetric with respect to the whole group $G$, and by means of the patterns seen in Fig. 1 the group structure is easily understood (note that neighboring vortices have opposite sense of rotation). The elements of $D_{4}$ correspond to rotations and reflections of the square, in some cases combined with a shift by $\pi / 4$ parallel to one of the boundaries. The subgroups $Z_{4}$ and $Z_{2}$, respectively, are generated by pure translations, for which the pattern remains invariant due to the periodicity.

In the following the bifurcations observed for increasing the forcing parameter $f$ as well as the resulting solution branches are described. An overview of the different solution branches is given in Table I and a schematic bifurcation diagram in Fig. 2.

\begin{tabular}{lll}
\hline \hline Branch & Stability interval & Remarks \\
\hline Steady I & $f<402.8$ & $8 \times 8$ vortex array \\
Cycles & $402.8<f<610.9$ & heteroclinic cycles \\
Chaos I & $610.9<f<679$ & Shilnikov-like chaos \\
Period I & $679<f<803.3$ & \\
Torus & $769.3<f<825.9$ & \\
Period II & $825.9<f<857.6$ & \\
Steady II & $857.6<f<961.7$ & large scale steady state \\
Period III & $961.7<f<1022.8$ & \\
Chaos II & $1022.8<f$ & \\
\hline \hline
\end{tabular}

TABLE I. The different solution branches. 


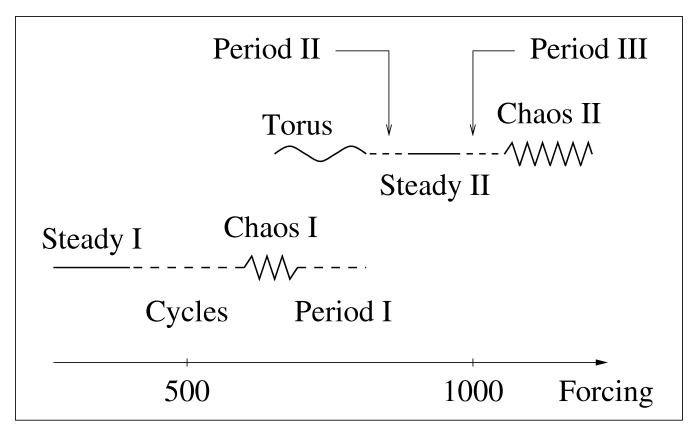

FIG. 2. Schematic bifurcation diagram

The primary steady state (Steady I, described above) loses its stability in a special bifurcation at $f=402.8$, where four real eigenvalues of the Jacobian matrix become zero. As a result of this symmetry breaking bifurcation an asymtotic heteroclinic cycle is born. This cycle consists of sixteen unstable fixed points (steady states), which are connected by their stable and unstable manifolds. A projection of the heteroclinic cycle onto the plane spanned by the real parts of two of the Fourier modes of $\omega$ is shown in Fig. 3.

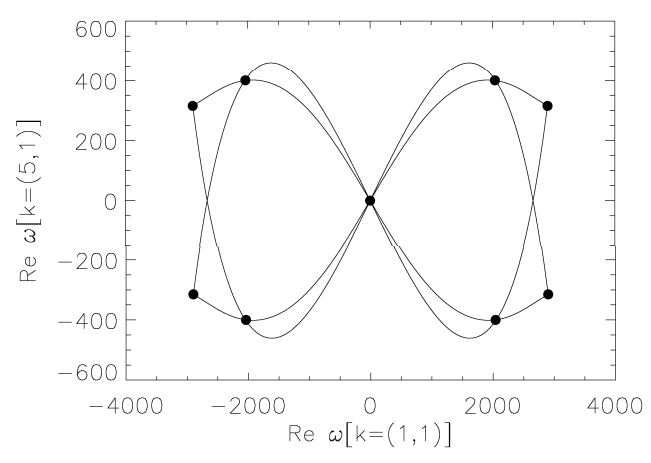

FIG. 3. Projection of the heteroclinic cycle for $f=475$. Filled circles mark the positions of fixed points.

The fixed points are marked by filled circles, some of them coinciding in the projection. In numerical simulations of the long-term behavior we observed the trajectory to stay a long time in the vicinity of one of the fixed points and then to switch rapidly to the next one, in the course of this slowly approaching the heteroclinic orbit. Theoretically the time period of the approximate cycle should go to infinity, but due to numerical errors it tyipically reached maximum values $T \approx 10^{4}$. In Fig. 4 for one of the fixed points in the cycle the velocity streamlines are shown. The original symmetry is partly broken. Symmetry breakings connected with the appearance of heteroclinic cycles are a typical phenomenon in systems with symmetry [18].

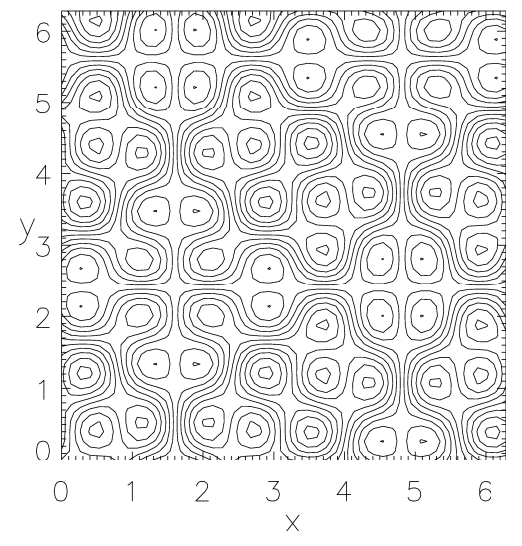

FIG. 4. Streamlines for $f=475$ (fixed point in the heteroclinic cycle)

The heteroclinic orbits connecting the fixed points dominate the dynamics of the system in a large parameter range. Then the cycle undergoes a complex bifurcation sequence, which we did not investigated in detail. The cycle seems to decay into several smaller heteroclinic cycles. At $f=610.9$ the system develops a chaotic dynamics (Chaos I); the calculation of the largest Lyapunov exponents yields one slightly positiv exponent. In Fig. 5 a projection of the trajectory for $f=611$ is shown. It is strongly indicative of a Shilnikov-type chaos. Also remnants of the heteroclinic cycle shown in Fig. 3 are still visible.

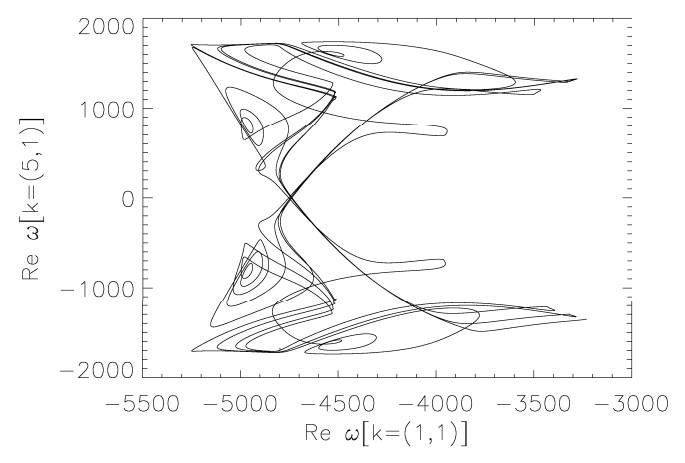

FIG. 5. Projection of the trajectory for $f=611$ (Chaos I).

This behavior is found up to $f=679$, where the dynamics of the chaotic attractor is replaced by a periodic motion (Period I). The topological structure of the corresponding streamline portrait is similar to that in Fig. 4. For increasing Reynolds number the basin of attraction of this periodic branch shrinks and finally disapperars for $f=803.3$.

As is seen in Fig. 2, for higher Reynolds numbers another main branch dominates the dynamics of the system. So we have found a further steady state (Steady II), stable within the parameter interval $857.6<f<961.7$ and 
corresponding to a qualitatively new solution structure in physical space. The velocity streamlines in Fig. 6 show that now motions on larger scales, comparable with the size of the system, become dominant and determine the spatial structure of the flow.

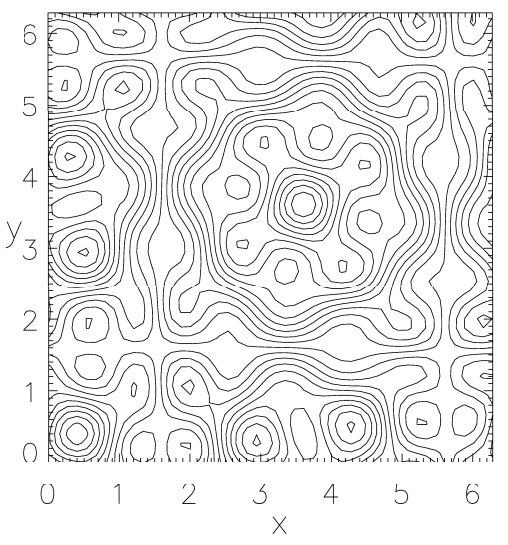

FIG. 6. Streamlines of the steady state found for $f=905$ (Steady II).

This branch was traced using continuation techniques, both for decreasing and for increasing Reynolds numbers. At both ends, at $f=857.6$ and $f=961.7$, Hopf bifurcations were observed, leading to periodic orbits, Period II and Period III. With a further decrease of $f$ a secondary Hopf bifurcation leads to a torus solution (Torus), which is stable only within a small parameter interval and coexists partly with the periodic branch Period I, which is characterized by small-scale spatial strucures.

When tracing the branch Period III towards higher Reynolds nummbers, chaotic motions are observed for $f>1022.8$ (branch Chaos II). The occurence of chaos has been verified by calculating the largest Lyapunov exponents for selected values of the bifurcation parameter, using an algorithm of Shimada and Nagashima [19]. Fig. 7 shows for $f=1041$ the cumulative values of the six largest Lyapunov exponents in dependence on the integration time. A good convergence is demonstrated, as well as that three of the exponents are positive.

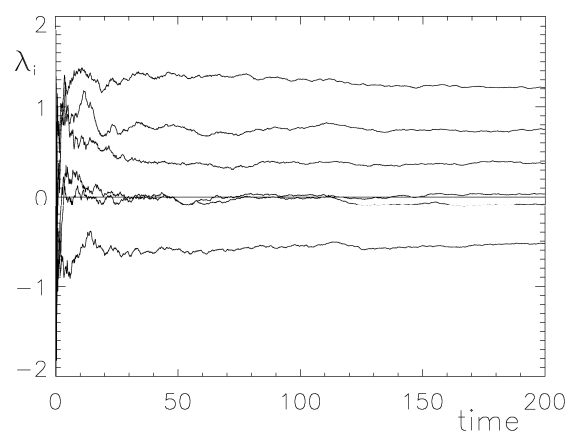

FIG. 7. The six largest Lyapunov exponents versus integration time for $f=1041$ (Chaos II).

One of the remaining open problems is the transition from the periodic branch Period III to chaos. We could not resolve a periodic-doubling cascade and numerical experiments indicated that in the vicinity of the transition point transient chaos occurs. For a value of the bifurcation parameter slighly below the transition value, a trajectory starting in the neighborhood of the chaotic set moves for a long time seeminly chaoticly and then suddenly settles down to a periodic orbit. Such a behavior can be caused by a crisis [20], but more detailed investigations, such as the determination of a scaling law for the transient time, are still under way.

\section{MIXING AND LAGRANGIAN TURBULENCE}

In the this section we try to distinguish between different dynamic regimes by means of tracers injected into the fluid, with the aim to make feasible a comparison of theoretical results with experimental observations.

For a steady flow the pathlines of tracers coincide with the streamlines and provide a direct visualization of the velocity field. In the time-dependent regimes the situation is much more complicated. The differential equations for the determination of the pathlines is now nonautonomous,

$$
\dot{\boldsymbol{x}}=\boldsymbol{v}(\boldsymbol{x}, t)
$$

for the normal space variables $\boldsymbol{x}$. It is known that already for simple periodic flows the pathlines can show a very complicated, wrinkled and chaotic form [21], a phenomenon also termed Lagrangian turbulence. The motion of tracers injected into the fluid in the form of a blob or a straight line is the subject of the theory of mixing and transport processes. Comprehensive overviews of this topic are given in the monograph of Ottino [22] and in a special issue of the journal Chaos, Soliton \& Fractals [23].

Mixing consists basically of the stretching and folding of fluid elements, quantified by the stretching rate and by the Hausdorf dimension, respectively. The stretching of an infinitesimal line element leads two the definition of the largest Lyapunov exponent. By contrast, we have studied the stretching properties of finite filaments. It is known that the stretching rates of finite line elements are generally larger than the largest Lyapunov exponent [24], but there is a qualitative coincidence in that an exponential or polynomial (e.g., linear) growth with time is always common to both quantities.

In practice we injected a straight line of tracer particles into the fluid and followed their time evolution by solving the NSE and Eq. (8) for the pathlines simultaneously. An adaptive refinement technique has been used to get 
an equidistant distribution of tracers on the line, limiting the maximum number of particles to $10^{5}$. Additionally, we calculated the length of the line in every timestep. These calculations were done for the large-scale steady state (Steady II, $f=905$ ), a periodic state (Period III, $f=996$ ), the torus solution (Torus, $f=792$ ) and a chaotic branch (Chaos II, $f=1041$ ). The initial line element has the length $l_{0}=0.3$ and has been placed near the center of the square.

Fig. 8 shows the shape of this line after simulation of a time interval $\Delta t=1.0$ and demonstrates the different mixing properties for the four branches.
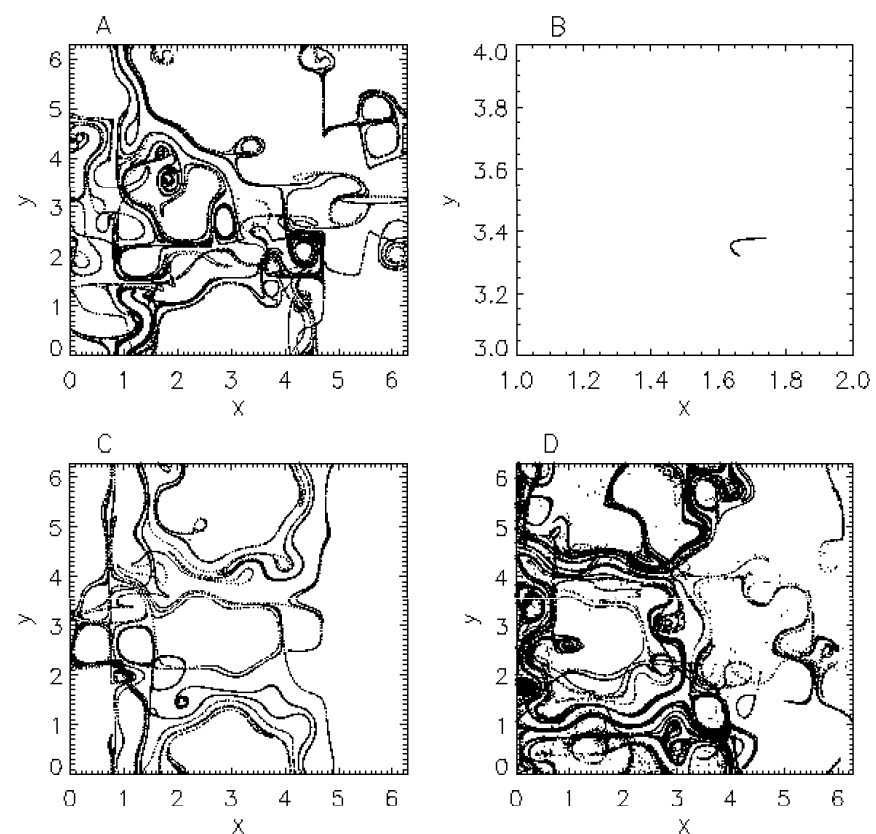

FIG. 8. The stretching of a line segment after a finite time in four different dynamical regimes $(\mathrm{A}=$ Torus, $\mathrm{B}=$ Steady II, $\mathrm{C}=$ Period III, D = Chaos II).

For the steady state the tracers have not been mixed and the line is only weakly streched due to the velocity gradient within an eddy. As espected, the mixing phenomenon becomes already visible in the periodic regime, but it is strongly enhanced for the quasiperiodic and for the chaotic motion. In a comparison of the quasiperiodic with the periodic regime it is remarkable that the mixing is much stronger the quasiperiodic case, though, due to a weaker forcing, the average velocity is much smaller (cf. Fig. 2).

In Fig. 9 the growth of the line element, a measure for the stretching rate, is presented for a simulated time interval of $\Delta t=2.0$.

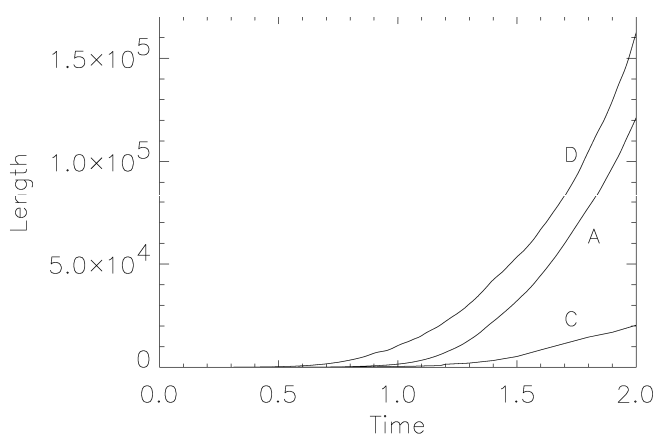

FIG. 9. The time evolution of the line length (the lettering corresponds to that used in Fig 8).

The stretching corresponding to the steady state is very small, so it has been droped here. It is recognizable that both the quasiperiodic and the chaotic motions lead to an exponential growth. In contrast, in the periodic regime the stretching effect is much weaker, somewhere in-betweeen a linear and an exponential growth.

To evaluate the fractal properies of the line element, supposed to result from its successive stretching and folding, we estimated its Hausdorff dimension by means of a fast implementation of a box-counting algorithm, which is based on a specific data structure [25]. For the time dependent velocity fields (Torus, Period III, Chaos II) the Hausdorff dimension of the injected filament increases with time, whereas it remains constant, $D_{H D}=1$, for the steady state. Table II shows the Hausdorff dimensions for the different dynamical regimes, calculated at $t=1.0$.

\begin{tabular}{c|cccc}
\hline \hline $\mathrm{f}$ & A: 792 & B: 905 & C: 996 & D: 1041 \\
\hline$D_{H D}$ & 1.58 & 1 & 1.38 & 1.66 \\
\hline \hline
\end{tabular}

TABLE II. The estimated Hausdorff dimensions at $t=1.0$ for the different regimes. 
It turns out that the Hausdorff dimension is nearly the same for both the torus and the chaotic branch, and is lower for the periodic solution. In general the differences in the values are not very significant and it seems that the Hausdorff dimension, calculated at an arbitrary time point, does not provide a reliable quantitative measure to distinguish between different dynamical regimes. It appears more promising to consider the time dependence of $D_{K Y}$ or to look for saturation values of $D_{K Y}$ after long times. But due to numerical limitations of the used algorithm as well as restricted computer time we have to shelve this question.

Concluding it can be stated that neither the length of line elements nor the Hausdorff dimension seem to be suitable measures for the distinction of dynamical regimes in a simple manner. The investigation of other measures will will be part of forthcoming work.

\section{DISCUSSION}

We have investigated the dynamics of a twodimensional fluid, for which an array of vortices is driven by an external forcing, and have analysed its bifurcations in the transition to chaos. Each physical system and in particular each hydrodynamical systems, shows its own specific bifurcations varying upon control parameters like the Reynolds number. For instance, in a special parameter region of the celebrated Lorenz system, representing a strongly truncation model of Reyleigh-Bénard convection, an infinite period doubling cascade leads to chaos [26]. Angelo and Riela [27], as well as Franceschini and Tebaldi [4] have found the same transition to chaos via period doubling bifurcations in low-mode truncations of the two-dimensional NSE with an external forcing of the Kolmogorov type. Another route to chaos via a quasiperiodic motion, in which an attracting torus loses its smothness, has been described by Franceschini in a generalization of this model including more modes [28]. In contrast to these scenarios, quasiperiodic solutions can also undergo an infinite sequence of torus doublings on their way to chaos if a different forcing in the NSE is used [9]. All these examples should demonstrate the variety of bifurcation scenarios in the transition to turbulence as well as that the behavior of each system has its specific character. We think, that the investigated bifurcation behavior of our system of driven vortices provides general insight into the dynamics of two-dimensional NavierStokes flows, which are used to model experiments as descibed in [10-14]. A correctly described feature is the appearence of long wave instabilities, in which scales much larger than that of the forcing are excited. These effects can be interpreted as a result of an inverse energy cascade, a typical property of two-dimensional NavierStokes flows. The same phenomenon was observed by Guzdar et al. [16] who studied a similar system, namely a linear array of forced vortices $(4 \times 1$ counterrotating vortices). These authors also observed the appearence of long wave lengths as result of an instability, which they called shear instability. A common feature of both systems is that at higher Reynolds numbers the large scales dominante the dynamics of the flow.

In conclusion we add few remarks on the role of the boundary conditions for the bifurcations and the character of the resulting solution branches. The periodic boundary conditions lead to a special symmetry group, given by Eq. (6), with respect to which the partial differential equations studied, Eqs. (1,2), are equivariant. Obviously the first bifurcations, leading to a heteroclinic cycle and afterwards to the appearance of Shilnikov-like chaos (see Fig. 5), are directly related to the imposed symmetry. Therefore, these phenomena cannot be expected to be observable in experiments like those mentioned in Sec. I. But we think that the nature of the solutions after the symmetry breaking, in particular of those belonging to the upper main branch in Fig. 2, characterized by large-scale spatial structures, are only little influenced by the boundary conditions and thus admit a physical interpretation. That the boundary conditions have only minor influence on the character of these solutions could be partially confirmed by preliminary test calculations with stress-free boundary conditions on the top and bottom boundaries and periodicity in the horizontal directions. Yet a more accurate study of the influence of different boundary conditions, including the role of bottom friction, present in the experiments and neglected in the this study, remains to be done and will be part of future investigations.

\section{ACKNOWLEDGEMENT}

We wish to thank Wolfgang Jansen, who put at our disposal the program to estimate the Hausdorff dimension by means of a fast box-counting algorithm. Furthermore, we acknowledge Sten Rüdiger, who helped us to find out the full symmetry group of the equations and last not least we thank the Max-Planck-Gesellschaft, which supported this research.

[1] L. D. Meshalkin and Y. G. Sinai, J. Appl. Math. Mech. 25, 1700 (1961).

[2] A. Thess, Phys. Fluids 4, 1385 (1992).

[3] C. Boldrighini and V. Franceschini, Commun. Math. Phys. 64, 159 (1979).

[4] V. Franceschini and C. Tebaldi, J. Stat. Phys. 21, 707 (1979).

[5] V. Franceschini, C. Giberti, and M. Nicolini, J. Stat. Phys. 50, 879 (1988). 
[6] V. Franceschini and C. Giberti, Theor. Comput. Fluid Dyn. 2, 185 (1991)

[7] J. Lee, Chaos 2, 537 (1992).

[8] F. Feudel and N. Seehafer, Chaos, Solitons \& Fractals 5, 1805 (1995).

[9] F. Feudel and N. Seehafer, Phys. Rev. E 52, 3506 (1995).

[10] J. Sommeria, J. Fluid Mech. 170, 139 (1986).

[11] P. Tabeling, B. Perrin, and S. Fauve, Europhys. Lett. 3, 459 (1987).

[12] P. Tabeling, O. Cardoso, and B. Perrin, J. Fluid Mech. 213, 511 (1990).

[13] O. Cardoso, H. Willaime, and P. Tabeling, Phys. Rev. Lett. 65, 1869 (1990).

[14] H. Willaime, O. Caedoso, and P. Tabeling, Phys. Rev. E 48, 288 (1993)

[15] J. M. Finn, J. F. Drake, and P. N. Guzdar, Phys. Fluids B 4, 2758 (1992).

[16] P. N. Guzdar, J. M. Finn, A. V. Rogalsky, and J. F. Drake, Phys. Rev. E 49, 2062 (1994).

[17] M. Golubitsky, I. Stewart, and D. G. Schaeffer, Singularities and Groups in Bifurcation Theory (Springer-Verlag, New York, 1988), Vol. II.

[18] M. Krupa, Robust heteroclinic cycles, to be published in J. Nonl. Sci.

[19] I. Shimada and T. Nagashima, Progr. Theor. Phys. 61, 1605 (1979).

[20] C. Grebogi, E. Ott, and J. A. Yorke, Physica D 7, 181 (1983).

[21] H. Aref, J. Fluid. Mech. 143, 1 (1984).

[22] J. M. Ottino, The Theory of Mixing: Stretching, Chaos and Transport (Cambridge Univ. Press, Cambridge, 1989).

[23] in Chaos Applied to Fluid Mixing, Vol. 4 of Chaos, Solitons $\& 3$ Fractals, edited by H. Aref (Pergamon Press, Oxford, 1994), pp. 745-1116.

[24] M. Tabor and I. Klapper, Chaos, Solitons \& Fractal 4, 1031 (1994).

[25] W. Jansen, Personal communication.

[26] E. A. Jackson, Perspectives of nonlinear dynamics (Cambridge University Press, Cambridge, 1994), Vol. 2.

[27] P. M. Angelo and G. Riela, Nouvo Cimento B 64, 207 (1981).

[28] V. Franceschini, Physica D 6, 285 (1983). 